\title{
Radiation Effect on Natural Convection near a Vertical Plate Embedded in Porous Medium with Ramped Wall Temperature
}

\author{
Sanatan Das, Mrinal Jana, Rabindra Nath Jana \\ Department of Applied Mathematics, Vidyasagar University, Midnapore, India \\ E-mail: jana261171@yahoo.co.in \\ Received November 16, 2011; revised December 7, 2011; accepted December 20, 2011
}

\begin{abstract}
Radiation effect on the natural convection flow of an optically thin viscous incompressible fluid near a vertical plate with ramped wall temperature in a porous medium has been studied. The exact solution of momentum and energy equations is obtained by the use of Laplace transform technique. The variations in fluid velocity and temperature are shown graphically whereas the numerical values of shear stress and the rate of heat transfer at the wall are presented in tabular form for various values of flow parameters. The results show that the fluid velocity increases with increase in Grashof number, Darcy number and time parameters whereas the fluid velocity decreases with increase in the radiation parameter and Prandtl number for ramped temperature as well as isothermal wall temperature. It is found that an increase in radiation parameter leads to rise the temperature for both ramped wall temperature as well as isothermal wall temperature. Further, it is found that an increase in Prandtl number leads to fall the temperature for both ramped wall temperature as well as isothermal wall temperature. The shear stress at the wall decreases with increases in either Prandtl number or porosity parameter while the result shows reverse in the case of radiation parameter. Finally, the rate of heat transfer is increased with increase in the radiation parameter for both ramped wall temperature as well as isothermal wall temperature.
\end{abstract}

Keywords: Natural Convection, Darcy Number, Radiation Parameter, Prandtl Number, Porous Medium, Ramped Wall Temperature and Isothermal Wall Temperature

\section{Introduction}

The phenomenon of natural convection arises in fluids when temperature changes cause density variations leading to buoyancy forces acting on the fluid particles. Such flows which are driven by temperature differences abound in nature and have been studied extensively because of its applications in engineering, geophysical and astrophysical environments. Comprehensive literature on various aspects of free convection flows and its applications could be found in Ghoshdastidar [1], Nield and Bejan [2]. Ghoshdastidar gave various areas of applications of free convection flow such as those found in heat transfer from pipes and transmission lines as well as from electronic devices, heat dissipation from the coil of a refrigerator unit to the surrounding air, heat transfer from a heater to room air, heat transfer in nuclear fuel rods to the surrounding coolant, heated and cooled en- closures, quenching, wire-drawing and extrusion, atmospheric and oceanic circulation. Unsteady free convection flows in a porous medium have received much attention in recent time due to its wide applications in geothermal and oil reservoir engineering as well as other geophysical and astrophysical studies. Moreover, considerable interest has been shown in radiation interaction with convection for heat and mass transfer in fluids. This is due to the significant role of thermal radiation in the surface heat transfer when convection heat transfer is small, particularly in free convection problems involving absorbing-emitting fluids. The unsteady fluid flow past a moving plate in the presence of free convection and radiation were studied by Mansure [3], Raptis and Perdikis [4], Das et al. [5], Grief et al. [6], Ganeasan and Loganathan [7], Mbeledogu et al. [8], Makinde [9] and Abdus-Sattar and Hamid Kalim [10]. All these studies have been confined to unsteady flow in a non-porous medium. 
Israel-Cookey et al. [11] have studied the influence of viscous dissipation and radiation on unsteady MHD freeconvection flow past an infinite heated vertical plate in a porous medium with time-dependent suction. Radiative and free convective effects of a MHD flow through a porous medium between infinite parallel plates with time dependent suction have been investigated by Alagoa et al. [12]. Israel-Cookey et al. [13] have made an analysis on MHD oscillatory Couette flow of a radiating viscous fluid in a porous medium with periodic wall temperature. Sattar and Maleque [14,15] have studied the unsteady MHD Natural convection flow and mass transfer along an accelerated porous plate in a porous medium. Thermal radiation interaction with unsteady MHD flow past a vertical porous plate immersed in a porous medium has been analyzed by Samad and Rahman [16]. Mahanti and Gaur [17] have studied the effects of varying viscosity and thermal conductivity on steady free convective flow and heat transfer along an isothermal vertical plate in the presence of heat sink. Transient free convection past a semi-infinite vertical plate with variable surface temperature has been investigated by Takhar et al. [18].

In this present paper, we investigate the effects of radiation on the free convection flow of an optically thin incompressible viscous fluid past an infinite vertical plate with ramped wall temperature in porous medium. The fluid considered is a gray, radiation, absorbing, emitting but non-scattering medium and the Rosseland approximation is used to describe the radiative heat transfer in the energy equation. It is seen that the velocity $u_{1}$ decreases for both ramped wall temperature as well as isothermal wall temperature with an increase in either radiation parameter $R a$ or Prandtl number $\mathrm{Pr}$. It is also seen that the velocity $u_{1}$ increases for both ramped wall temperature as well as isothermal wall temperature with an increase in either Grashof number $\mathrm{Gr}$ or time $\tau$. It is found that an increase in radiation parameter $R a$ leads to rise the temperature $\theta$ for both ramped wall temperature as well as isothermal wall temperature. Further, it is found that an increase in Prandtl number leads to fall the temperature for ramped temperature as well as isothermal case.

\section{Formulation of the Problem and Its Solutions}

Consider the unsteady free convection flow of an optically thin viscous incompressible fluid past an moving infinite vertical plate coinciding with plane $y=0$, where the flow is confined to $y>0$ in a porous medium. Choose a cartesian co-ordinates system with $x$-axis along the wall in a vertically upward direction and $y$-axis is normal to it into the fluid (see Figure 1). At $t \leq 0$, the

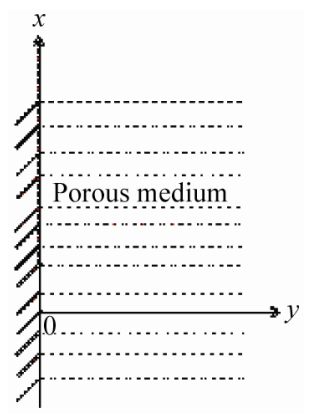

Figure 1. Geometry of the problem.

plate and the surrounding fluid are at the same constant temperature $T_{\infty}$. At time $t>0$, the temperature of the wall is raised or lowered to $T_{\infty}+\left(T_{w}-T_{\infty}\right) \frac{t}{t_{0}}$ when

$0<t \leq t_{0}$ and the constant temperature $T_{w}$ is maintained at $t>t_{0}$. Since the plate is infinite along $x$-direction, all the physical variables are the function of $y$ and $t$ only. The flow is considered optically thin gray gas with natural convection and radiation. The radiative heat flux in the $x$-direction is considered negligible in comparison to $y$-direction.

The Boussinesq approximation is assumed to hold and for the evaluation of the gravitational body force, the density is assumed to depend on the temperature according to the equation of reference state

$$
\rho=\rho_{0}\left[1-\beta^{*}\left(T-T_{\infty}\right)\right],
$$

where $T$ is the fluid temperature, $\rho$ the fluid density, $\beta^{*}$ the coefficient of thermal expansion and $T_{\infty}$ and $\rho_{0}$ being the reference temperature and the density respectively.

Using Boussinesq Approximation (1), the momentum equation in a porous medium along $x$-axis is

$$
\frac{\partial u}{\partial t}=v \frac{\partial^{2} u}{\partial y^{2}}+g \beta^{*}\left(T-T_{\infty}\right)-\frac{v}{k^{*}} u,
$$

where $u, g, \beta^{*}, v, \rho$ and $k^{*}$ are respectively, fluid velocity, acceleration due to gravity, coefficient of thermal expansion, kinematic viscosity, fluid density and permeability of a porous media.

The energy equation is

$$
\frac{\partial T}{\partial t}=\frac{k}{\rho c_{p}} \frac{\partial^{2} T}{\partial y^{2}}-\frac{1}{\rho c_{p}} \frac{\partial q_{r}}{\partial y},
$$

where $k$ is the thermal conductivity, $c_{p}$ the specific heat at constant pressure and $q_{r}$ the radiative heat flux.

The initial and boundary conditions are

$$
\begin{gathered}
u=0, T=T_{\infty} \text { for } y \geq 0 \text { and } t \leq 0, \\
u=U_{0} \text { at } y=0 \text { for } t>0,
\end{gathered}
$$




$$
\begin{array}{ll}
T=T_{\infty}+\left(T_{w}-T_{\infty}\right) \frac{t}{t_{0}} & \text { at } y=0 \text { for } 0<t \leq t_{0} \\
T=T_{w} & \text { at } y=0 \text { for } t>t_{0} \\
u \rightarrow 0, T \rightarrow T_{\infty} & \text { as } y \rightarrow \infty \text { for } t>0
\end{array}
$$

It has been shown by Cogley et al. [19] that in the optically thin limit for a non-gray gas near equilibrium, the following relation holds

$$
\frac{\partial q_{r}}{\partial y}=4\left(T-T_{\infty}\right) \int_{0}^{\infty} K_{\lambda_{0}}\left(\frac{\partial e_{\lambda h}}{\partial T}\right)_{0} \mathrm{~d} \lambda,
$$

where $K_{\lambda}$ is the absorption coefficient, $\lambda$ is the wave length, $e_{\lambda h}$ is the Plank's function and subscript ' 0 ' indicates that all quantities have been evaluated at the temperature $T_{\infty}$ which is the temperature of the wall at time $t \leq 0$. Thus our study will be limited to small difference of wall temperature to the fluid temperature.

On the use of (5), Equation (3) becomes

$$
\frac{\partial T}{\partial t}=\frac{k}{\rho c_{p}} \frac{\partial^{2} T}{\partial y^{2}}-\frac{4}{\rho c_{p}}\left(T-T_{\infty}\right) I,
$$

where

$$
I=\int_{0}^{\infty} K_{\lambda_{0}}\left(\frac{\partial e_{\lambda h}}{\partial T}\right)_{0} \mathrm{~d} \lambda .
$$

Introducing dimensionless variables

$$
\eta=\frac{y}{U_{0} t_{0}}, \quad \tau=\frac{t}{t_{0}}, \quad u_{1}=\frac{u}{U_{0}}, \quad \theta=\frac{T-T_{\infty}}{T_{w}-T_{\infty}},
$$

Equations (2) and (6) become

$$
\begin{gathered}
\frac{\partial u_{1}}{\partial \tau}=\frac{\partial^{2} u_{1}}{\partial \eta^{2}}-\frac{1}{\sigma} u_{1}+G r \theta, \\
\frac{\partial \theta}{\partial \tau}=\frac{1}{\operatorname{Pr}} \frac{\partial^{2} \theta}{\partial \eta^{2}}-R a \theta,
\end{gathered}
$$

where $G r=\frac{g \beta^{*} v\left(T_{w}-T_{\infty}\right)}{U_{0}^{3}}$ is Grashof number, $\operatorname{Pr}=\frac{v \rho c_{p}}{k}$ the Prandtl number, $R a=\frac{4 I v}{\rho c_{p} U_{0}^{2}}$ the radiation parameter, $\sigma=\frac{k^{*} U_{0}^{2}}{v^{2}}=M a D a$ the porosity parameter and $D a$ the Darcy number.

The characteristic time $t_{0}$ is defined as

$$
t_{0}=\frac{v}{U_{0}^{2}} .
$$

The corresponding initial and boundary conditions for $u_{1}$ and $\theta$ are

$$
u_{1}=0, \theta=0 \text { for } \eta \geq 0 \text { and } \tau \leq 0,
$$

$$
\begin{gathered}
u_{1}=1 \text { at } \eta=0 \text { for } \tau>0, \\
\theta=\tau \text { at } \eta=0 \text { for } 0<\tau \leq 1, \\
\theta=1 \text { at } \eta=0 \text { for } \tau>1, u_{1} \rightarrow 0, \\
\theta \rightarrow 0 \text { as } \eta \rightarrow \infty \text { for } \tau>0 .
\end{gathered}
$$

Taking Laplace transformation of the Equations (9) and (10), we get

$$
\begin{aligned}
\frac{\mathrm{d}^{2} \bar{u}_{1}}{\mathrm{~d} \eta^{2}}-\left(s+\frac{1}{M a D a}\right) \bar{u}_{1} & =-G r \bar{\theta}, \\
\frac{\mathrm{d}^{2} \bar{\theta}}{\mathrm{d} \eta^{2}}-\operatorname{Pr}(s+R a) \bar{\theta} & =0,
\end{aligned}
$$

where

$$
\begin{aligned}
& \bar{u}_{1}(\eta, s)=\int_{0}^{\infty} u_{1}(\eta, \tau) e^{-s \tau} \mathrm{d} \tau, \\
& \bar{\theta}(\eta, s)=\int_{0}^{\infty} \theta(\eta, \tau) e^{-s \tau} \mathrm{d} \tau .
\end{aligned}
$$

The corresponding boundary conditions for $\bar{u}_{1}$ and $\bar{\theta}$ are

$$
\begin{aligned}
& \bar{u}_{1}=\frac{1}{s}, \bar{\theta}=\frac{1}{s^{2}}\left(1-e^{-s}\right) \text { at } \eta=0, \\
& \bar{u}_{1} \rightarrow 0, \bar{\theta} \rightarrow 0 \text { as } \eta \rightarrow \infty .
\end{aligned}
$$

The solution of the Equations (14) and (13) subject to the boundary conditions (16) can be easily obtained and are given by

$$
\bar{\theta}(\eta, s)=\frac{\left(1-e^{-s}\right)}{s^{2}} e^{-\eta \sqrt{\operatorname{Pr}(s+R a)}},
$$

$$
\begin{aligned}
\bar{u}_{1}(\eta, s)= & \frac{1}{s} e^{-\eta \sqrt{s+\frac{1}{M a D a}}} \\
& -\frac{\alpha\left(1-e^{-s}\right)}{s^{2}(s-\beta)}\left[e^{-\eta \sqrt{s+\frac{1}{M a D a}}}-e^{-\eta \sqrt{\operatorname{Pr}(s+R a)}}\right]
\end{aligned}
$$

where

$$
\alpha=\frac{G r}{(1-\operatorname{Pr})} \text { and } \beta=\frac{\left(\operatorname{Pr} R a-\frac{1}{M a D a}\right)}{(1-\operatorname{Pr})} .
$$

Taking the inverse Laplace transform of Equations (17) and (18), the solution for the fluid temperature $\theta(\eta, \tau)$ and fluid velocity $u_{1}(\eta, \tau)$ are obtained and are given by

$$
\begin{aligned}
\theta(\eta, \tau)= & \theta_{1}(\eta, \tau)-\theta_{1}(\eta, \tau-1) H(\tau-1) \\
u_{1}(\eta, \tau)= & \frac{1}{2}\left[e^{\frac{\eta}{\sqrt{M a D a}}} \operatorname{erfc}\left(\frac{\eta}{2 \sqrt{\tau}}+\sqrt{\frac{\tau}{M a D a}}\right)\right. \\
& \left.+e^{-\frac{\eta}{\sqrt{M a D a}}} \operatorname{erfc}\left(\frac{\eta}{2 \sqrt{\tau}}-\sqrt{\frac{\tau}{M a D a}}\right)\right]
\end{aligned}
$$




$$
-\alpha\left[\phi_{1}(\eta, \tau)-\phi_{1}(\eta, \tau-1) H(\tau-1)\right],
$$

where

$$
\begin{aligned}
& \theta_{1}(\eta, \tau)=\frac{1}{2}\left[\left(\tau+\frac{\eta}{2} \sqrt{\frac{\operatorname{Pr}}{R a}}\right) e^{\eta \sqrt{\operatorname{Pr} R a}}\right. \\
& \times \operatorname{erfc}\left(\frac{\eta}{2} \sqrt{\frac{\operatorname{Pr}}{\tau}}+\sqrt{R a \tau}\right) \\
& \left.+\left(\tau-\frac{\eta}{2} \sqrt{\frac{\operatorname{Pr}}{R a}}\right) e^{-\eta \sqrt{\operatorname{Pr} R a}} \operatorname{erfc}\left(\frac{\eta}{2} \sqrt{\frac{\operatorname{Pr}}{\tau}}-\sqrt{R a \tau}\right)\right], \\
& \phi_{1}(\eta, \tau)=\frac{1}{2}\left[\frac { e ^ { \beta \tau } } { \beta ^ { 2 } } \left\{e^{\eta \sqrt{\frac{1}{\mathrm{MaDa}}+\beta}}\right.\right. \\
& \times \operatorname{erfc}\left(\frac{\eta}{2 \sqrt{\tau}}+\sqrt{\left(\frac{1}{M a D a}+\beta\right) \tau}\right) \\
& +e^{-\eta \sqrt{\frac{1}{M a D a}+\beta}} \operatorname{erfc}\left(\frac{\eta}{2 \sqrt{\tau}}-\sqrt{\left(\frac{1}{M a D a}+\beta\right) \tau}\right) \\
& -e^{\eta \sqrt{\operatorname{Pr}(R a+\beta)}} \operatorname{erfc}\left(\frac{\eta}{2} \sqrt{\frac{\operatorname{Pr}}{\tau}}+\sqrt{(R a+\beta) \tau}\right) \\
& \left.-e^{-\eta \sqrt{\operatorname{Pr}(R a+\beta)}} \operatorname{erfc}\left(\frac{\eta}{2} \sqrt{\frac{\operatorname{Pr}}{\tau}}-\sqrt{(R a+\beta) \tau}\right)\right\} \\
& -\frac{1}{\beta}\left(\tau+\frac{1}{\beta}+\frac{\eta \sqrt{M a D a}}{2}\right) e^{\frac{\eta}{\sqrt{M a D a}}} \\
& \times \operatorname{erfc}\left(\frac{\eta}{2 \sqrt{\tau}}+\sqrt{\frac{\tau}{M a D a}}\right) \\
& -\frac{1}{\beta}\left(\tau+\frac{1}{\beta}-\frac{\eta \sqrt{M a D a}}{2}\right) e^{-\frac{\eta}{\sqrt{M a D a}}} \\
& \times \operatorname{erfc}\left(\frac{\eta}{2 \sqrt{\tau}}-\sqrt{\frac{\tau}{M a D a}}\right)+\frac{1}{\beta}\left(\tau+\frac{1}{\beta}+\frac{\eta}{2} \sqrt{\frac{\operatorname{Pr}}{R a}}\right) e^{\eta \sqrt{\operatorname{Pr} R a}} \\
& \times \operatorname{erfc}\left(\frac{\eta}{2} \sqrt{\frac{\operatorname{Pr}}{\tau}}+\sqrt{R a \tau}\right)+\frac{1}{\beta}\left(\tau+\frac{1}{\beta}-\frac{\eta}{2} \sqrt{\frac{\operatorname{Pr}}{R a}}\right) \\
& \left.\times e^{-\eta \sqrt{\operatorname{Pr} R a}} \operatorname{erfc}\left(\frac{\eta}{2} \sqrt{\frac{\operatorname{Pr}}{\tau}}-\sqrt{\operatorname{Ra} \tau}\right)\right] \text {, }
\end{aligned}
$$

case $\operatorname{Pr}=1$ corresponds to those fluids for which both viscous and thermal boundary layer thickness are of the same order of magnitude. Setting $\operatorname{Pr}=1$ in Equation (14) and following the same procedure as before, the exact solution for the fluid temperature $\theta(\eta, \tau)$ and fluid velocity $u_{1}(\eta, \tau)$ is obtained and is expressed in the following form

$$
\begin{aligned}
& \theta(\eta, \tau)=\theta_{2}(\eta, \tau)-\theta_{2}(\eta, \tau-1) H(\tau-1), \\
& u_{1}(\eta, \tau)= \frac{1}{2}\left[e^{\frac{\eta}{\sqrt{M a D a}}} \operatorname{erfc}\left(\frac{\eta}{2 \sqrt{\tau}}+\sqrt{\frac{\tau}{M a D a}}\right)\right. \\
&\left.+e^{-\frac{\eta}{\sqrt{M a D a}}} \operatorname{erfc}\left(\frac{\eta}{2 \sqrt{\tau}}-\sqrt{\frac{\tau}{M a D a}}\right)\right] \\
&-\gamma\left[\phi_{2}(\eta, \tau)-\phi_{2}(\eta, \tau-1) H(\tau-1)\right],
\end{aligned}
$$

where

$$
\begin{aligned}
\theta_{2}(\eta, \tau) & =\frac{1}{2}\left[\left(\tau+\frac{\eta}{2 \sqrt{R a}}\right) e^{\eta \sqrt{R a}} \operatorname{erfc}\left(\frac{\eta}{2 \sqrt{\tau}}+\sqrt{R a \tau}\right)\right. \\
& \left.+\left(\tau-\frac{\eta}{2 \sqrt{R a}}\right) e^{-\eta \sqrt{R a}} \operatorname{erfc}\left(\frac{\eta}{2 \sqrt{\tau}}-\sqrt{R a \tau}\right)\right], \\
\phi_{2}(\eta, \tau)= & \frac{1}{2}\left[\left(\tau+\frac{\eta}{2 \sqrt{R a}}\right) e^{\eta \sqrt{R a}} \operatorname{erfc}\left(\frac{\eta}{2 \sqrt{\tau}}+\sqrt{R a \tau}\right)\right. \\
+ & \left(\tau-\frac{\eta}{2 \sqrt{R a}}\right) e^{-\eta \sqrt{R a}} \operatorname{erfc}\left(\frac{\eta}{2 \sqrt{\tau}}-\sqrt{R a \tau}\right) \\
& -\left(\tau+\frac{\eta \sqrt{M a D a}}{2}\right) e^{\frac{\eta}{\sqrt{M a D a}}} \operatorname{erfc}\left(\frac{\eta}{2 \sqrt{\tau}}+\sqrt{\frac{\tau}{M a D a}}\right) \\
& \left.-\left(\tau-\frac{\eta \sqrt{M a D a}}{2}\right) e^{-\frac{\eta}{\sqrt{M a D a}}} \operatorname{erfc}\left(\frac{\eta}{2 \sqrt{\tau}}-\sqrt{\frac{\tau}{M a D a}}\right)\right], \\
\text { and } \gamma= & G r /\left(R a-\frac{1}{M a D a}\right) .
\end{aligned}
$$

\subsection{Solution for Isothermal Case}

In order to highlight the effects of the ramped temperature distribution near a vertical plate, it may be important to compare the effects of the isothermal temperature distribution for the fluid flow. The temperature and the velocity for the fluid flow near an isothermal plate can be expressed as

$$
\begin{aligned}
\theta(\eta, \tau)= & \frac{1}{2}\left[e^{\eta \sqrt{\operatorname{Pr} R a}} \operatorname{erfc}\left(\frac{\eta}{2} \sqrt{\frac{\operatorname{Pr}}{\tau}}+\sqrt{\operatorname{Ra} \tau}\right)\right. \\
& \left.+e^{-\eta \sqrt{\operatorname{Pr} R a}} \operatorname{erfc}\left(\frac{\eta}{2} \sqrt{\frac{\operatorname{Pr}}{\tau}}-\sqrt{R a \tau}\right)\right],
\end{aligned}
$$

Prandtl number is a measure of the relative strength of the viscosity and thermal conductivity of the fluid. So the 


$$
\begin{aligned}
u_{1}(\eta, \tau)= & {\left[\frac{1}{2} e^{\frac{\eta}{\sqrt{M a D a}}} \operatorname{erfc}\left(\frac{\eta}{2 \sqrt{\tau}}+\sqrt{\frac{\tau}{M a D a}}\right)\right.} \\
& \left.+e^{-\frac{\eta}{\sqrt{M a D a}}} \operatorname{erfc}\left(\frac{\eta}{2 \sqrt{\tau}}-\sqrt{\frac{\tau}{M a D a}}\right)\right] \\
& -\alpha\left[u_{11}(\eta, \tau)-u_{12}(\eta, \tau)\right]
\end{aligned}
$$

where

$$
\begin{aligned}
& u_{11}(\eta, \tau)=\frac{1}{2 \beta}\left[e ^ { \beta \tau } \left\{e^{\eta \sqrt{\beta+\frac{1}{M a D a}}}\right.\right. \\
& \times \operatorname{erfc}\left(\frac{\eta}{2 \sqrt{\tau}}+\sqrt{\left(\beta+\frac{1}{M a D a}\right) \tau}\right) \\
& \left.+e^{-\eta \sqrt{\beta+\frac{1}{M a D a}}} \operatorname{erfc}\left(\frac{\eta}{2 \sqrt{\tau}}-\sqrt{\left(\beta+\frac{1}{M a D a}\right) \tau}\right)\right\} \\
& -\left\{e^{\frac{\eta}{\sqrt{M a D a}}} \operatorname{erfc}\left(\frac{\eta}{2 \sqrt{\tau}}+\sqrt{\frac{\tau}{M a D a}}\right)\right. \\
& \left.\left.+e^{-\frac{\eta}{\sqrt{M a D a}}} \operatorname{erfc}\left(\frac{\eta}{2 \sqrt{\tau}}-\sqrt{\frac{\tau}{M a D a}}\right)\right\}\right] \\
& u_{12}(\eta, \tau)=\frac{1}{2 \beta}\left[e ^ { \beta \tau } \left\{e^{\eta \sqrt{(\beta+R a) \operatorname{Pr}}}\right.\right. \\
& \times \operatorname{erfc}\left(\frac{\eta}{2} \sqrt{\frac{\operatorname{Pr}}{\tau}}+\sqrt{(\beta+R a) \tau}\right) \\
& \left.+e^{-\eta \sqrt{(\beta+R a) \operatorname{Pr}}} \operatorname{erfc}\left(\frac{\eta}{2} \sqrt{\frac{\operatorname{Pr}}{\tau}}-\sqrt{(\beta+R a) \tau}\right)\right\} \\
& -\left\{e^{\eta \sqrt{\operatorname{Pr} R a}} \operatorname{erfc}\left(\frac{\eta}{2} \sqrt{\frac{\operatorname{Pr}}{\tau}}+\sqrt{\operatorname{Ra} \tau}\right)\right. \\
& \left.\left.+e^{-\eta \sqrt{\operatorname{Pr} R a}} \operatorname{erfc}\left(\frac{\eta}{2} \sqrt{\frac{\operatorname{Pr}}{\tau}}-\sqrt{R a \tau}\right)\right\}\right] .
\end{aligned}
$$

When $\operatorname{Pr}=1$, the Solutions (27) and (28) become

$$
\begin{gathered}
\theta(\eta, \tau)=\frac{1}{2}\left[e^{\eta \sqrt{R a}} \operatorname{erfc}\left(\frac{\eta}{2 \sqrt{\tau}}+\sqrt{R a \tau}\right)\right. \\
\left.+e^{-\eta \sqrt{R a}} \operatorname{erfc}\left(\frac{\eta}{2 \sqrt{\tau}}-\sqrt{R a \tau}\right)\right], \\
u_{1}(\eta, \tau)=u_{13}(\eta, \tau)+\gamma\left[u_{13}(\eta, \tau)-u_{14}(\eta, \tau)\right],
\end{gathered}
$$

where

$$
u_{13}(\eta, \tau)=\frac{1}{2}\left[e^{\frac{\eta}{\sqrt{M a D a}}} \operatorname{erfc}\left(\frac{\eta}{2 \sqrt{\tau}}+\sqrt{\frac{\tau}{M a D a}}\right)\right.
$$

$$
\begin{array}{r}
\left.+e^{-\frac{\eta}{\sqrt{M a D a}}} \operatorname{erfc}\left(\frac{\eta}{2 \sqrt{\tau}}-\sqrt{\frac{\tau}{M a D a}}\right)\right], \\
u_{14}(\eta, \tau)=\frac{1}{2}\left[e^{\eta \sqrt{R a}} \operatorname{erfc}\left(\frac{\eta}{2 \sqrt{\tau}}+\sqrt{R a \tau}\right)\right. \\
\left.+e^{-\eta \sqrt{R a}} \operatorname{erfc}\left(\frac{\eta}{2 \sqrt{\tau}}-\sqrt{R a \tau}\right)\right] .
\end{array}
$$

\section{Results and Discussion}

We have plotted the non-dimensional velocity and temperature for several values of radiation parameter $R a$, Prandtl number $\mathrm{Pr}$, Grashof number $\mathrm{Gr}$, Darcy number $D a$ and time $\tau$ in Figures 2-9. Figures 2-6 represent the velocity $u_{1}$ against $\eta$ for several values of $R a, \operatorname{Pr}, G r, D a$ and $\tau$. Figure 2 shows that an increase in the radiation parameter $R a$ leads to fall in the velocity $u_{1}$ for both ramped wall temperature as well as isothermal wall temperature. Figure $\mathbf{3}$ displays that the velocity $u_{1}$ decreases for both ramped wall temperature as well as isothermal wall temperature with an increase in Prandtl number $\operatorname{Pr}$. Physically, this is true because the increase in the Prandtl number is due to increase in the viscosity of the fluid which makes the fluid thick and hence causes a decrease in the velocity of the fluid. It is observed from Figure 4 that an increase in $G r$, leads to a rise in the values of velocity $u_{1}$ due to enhancement in buoyancy force. Figure 5 reveals that the velocity $u_{1}$ increases for both ramped wall temperature as well as isothermal wall temperature with an increase in Darcy number $D a$. It is seen from Figure 6 that the velocity $u_{1}$ increases for both ramped wall temperature as well as isothermal wall temperature with an increase in time $\tau$. It is observed from Figure 7 that the temperature $\theta$ decreases as the radiation parameter $R a$ increases for both ramped wall temperature as well as isothermal wall temperature. This result qualitatively agrees with expectations, since the effect of radiation is to decrease the rate of energy transport to the fluid, thereby decreasing the temperature of the fluid. It is seen from Figure 8 that the temperature $\theta$ decreases for both ramped wall temperature as well as isothermal wall temperature with an increase in Prandtl number $\mathrm{Pr}$. This implies that an increase in Prandtl number leads to fall the thermal boundary layer flow for ramped temperature as well as isothermal wall temperature. The effect of the Prandtl number is very important in the temperature field. A fall in temperature occurs due to an increasing value of the Prandtl number. This is in agreement with the physical fact that the thermal boundary layer thickness decreases with increase in $\operatorname{Pr}$. Figure 9 


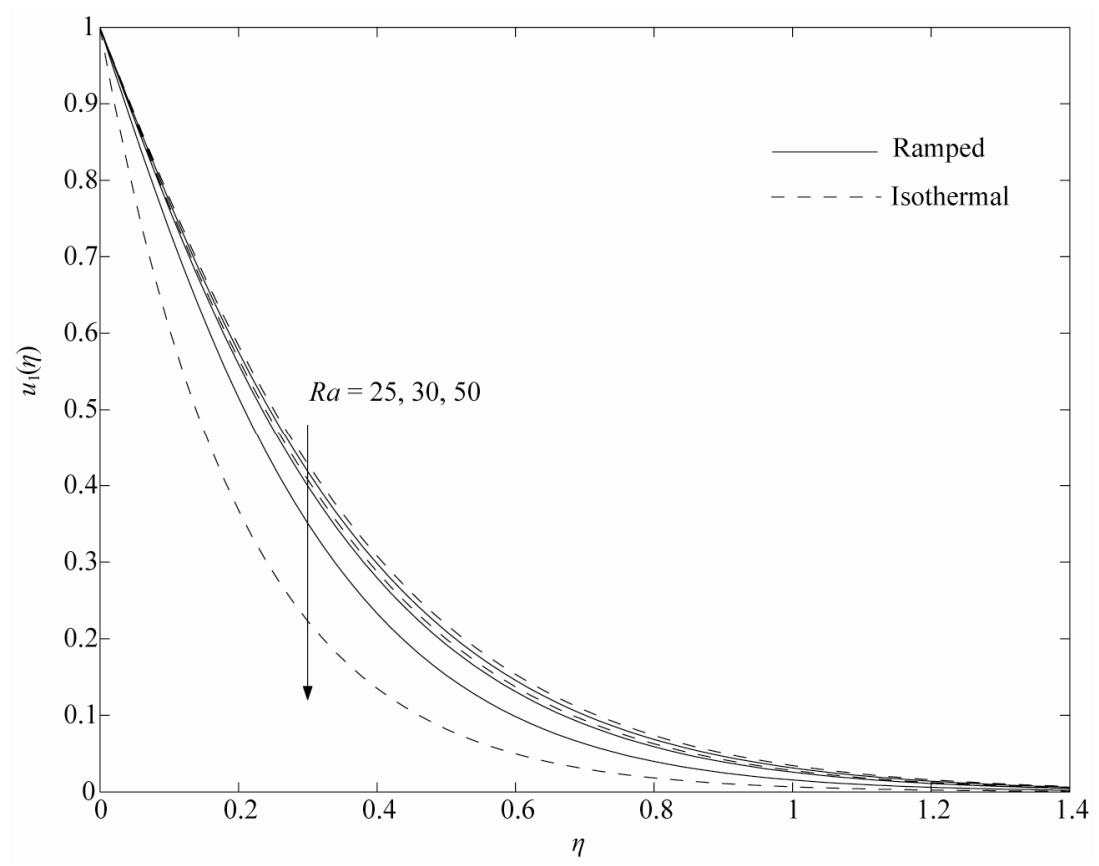

Figure 2. Velocity profiles for variations in $R a$ when $\operatorname{Pr}=0.71, G r=25, \tau=0.1$ and $D a=0.04$.

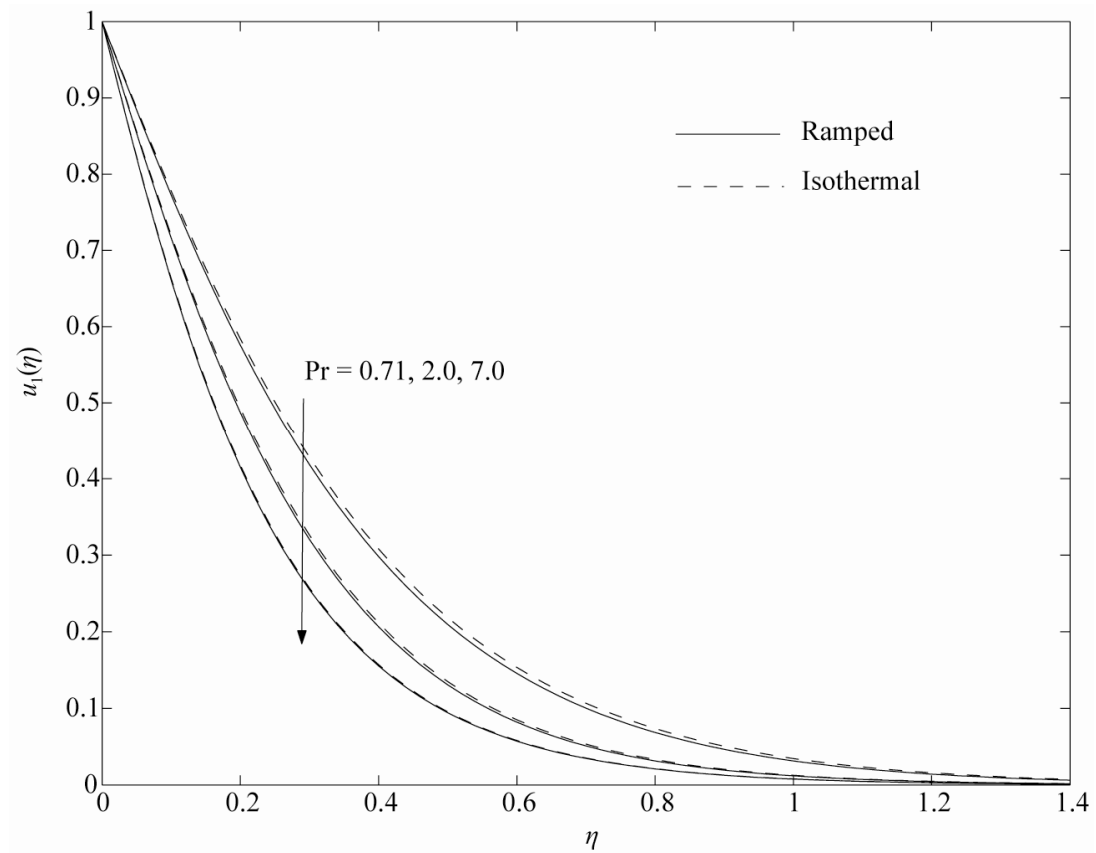

Figure 3. Velocity profiles for variations in $\operatorname{Pr}$ when $D a=0.04, G r=25, \tau=0.1$ and $R a=2$.

shows that the temperature $\theta$ increases for both ramped wall temperature as well as isothermal wall temperature with an increase in time $\tau$.

From the physical point of view, it is necessary to know the shear stress and the rate of heat transfer (or the Nusselt number) at the wall $(\eta=0)$. We have presented the expression for the rate of heat transfer $\mathrm{Nu}$ and shear stress $\tau_{0}$ at the wall $\eta=0$ in the following form for both the ramped wall temperature and isothermal wall temperature.

For the ramped wall temperature

$$
\left.N u=-\frac{\partial \theta}{\partial \eta}\right]_{\eta=0}=\theta_{3}(\eta, \tau)-\theta_{3}(\eta, \tau-1) H(\eta-1),
$$




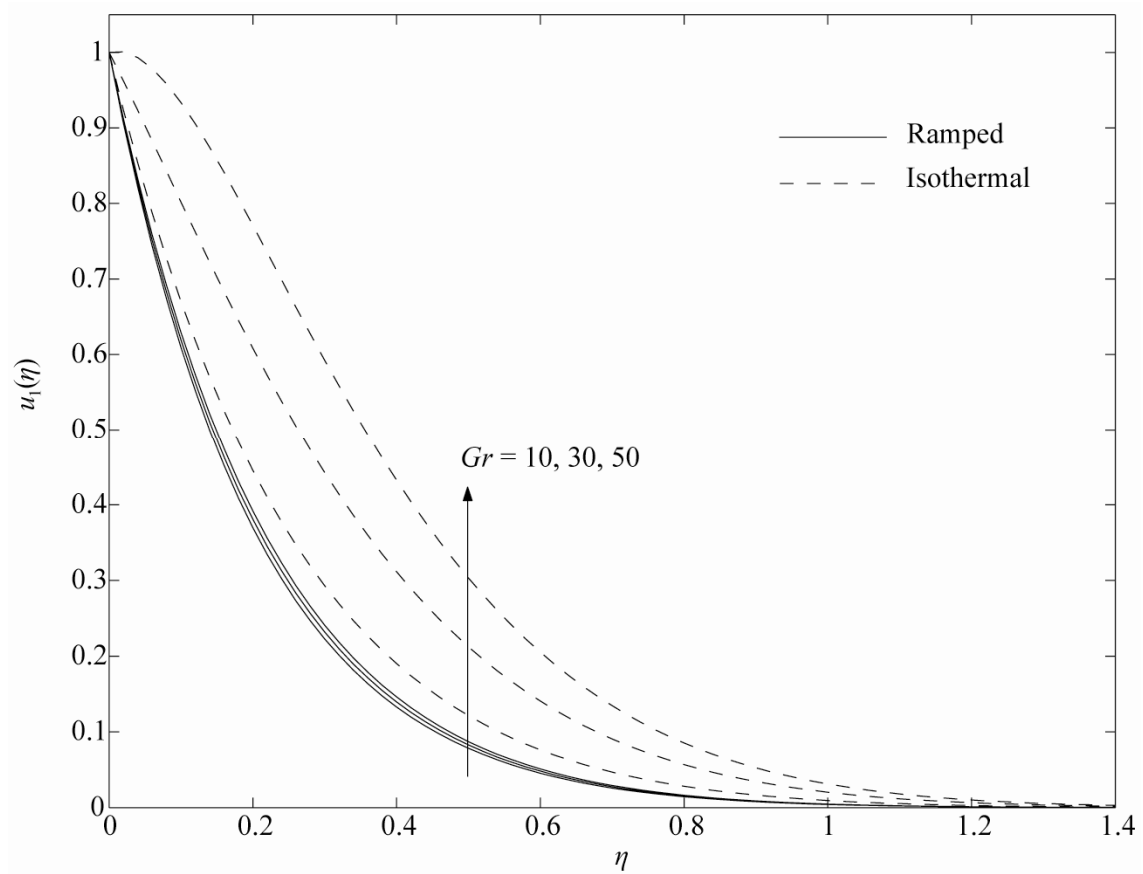

Figure 4. Velocity profiles for variations in $G r$ when $\operatorname{Pr}=0.71, D a=0.04, \tau=0.1$ and $R a=2$.

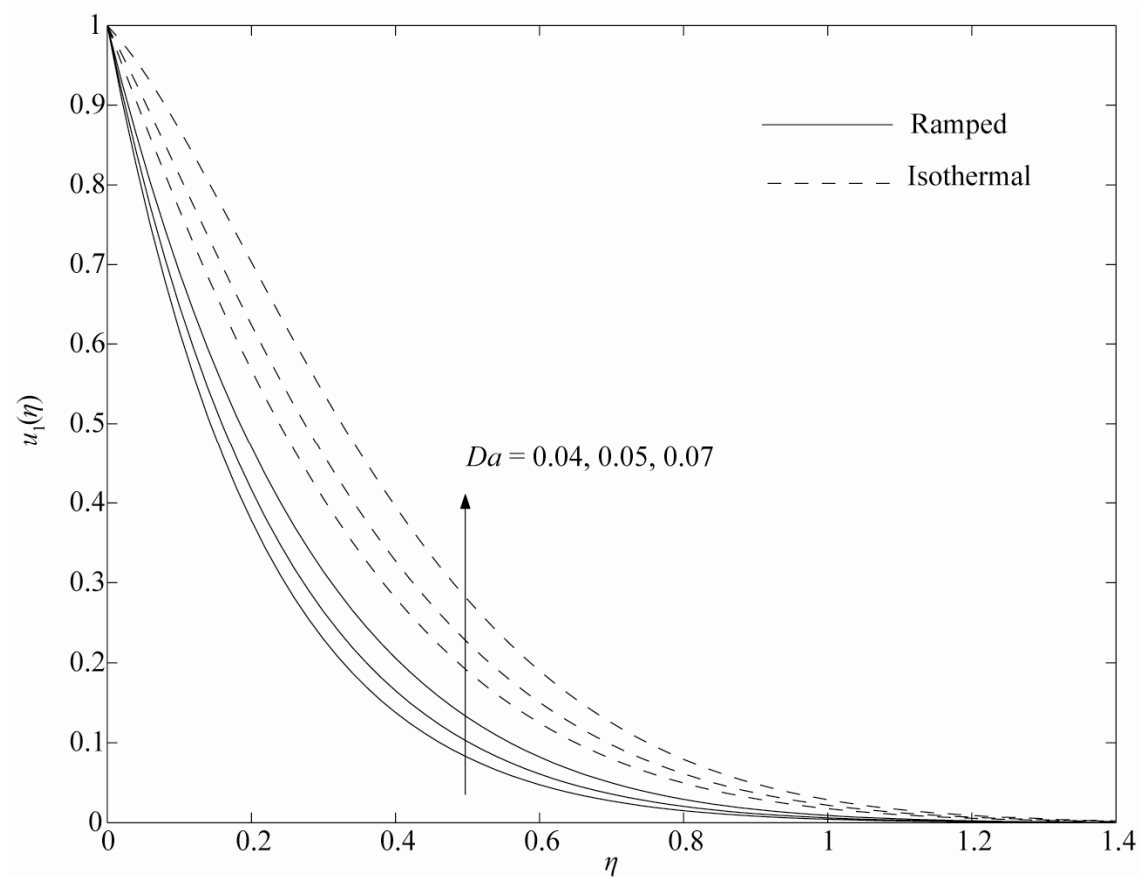

Figure 5. Velocity profiles for variations in $D a$ when $\operatorname{Pr}=0.71, G r=25, \tau=0.1$ and $R a=2$.

$$
\begin{aligned}
\tau_{0}= & \left.\frac{\partial u_{1}}{\partial \eta}\right]_{\eta=0}=-\left[\frac{1}{\sqrt{\pi \tau}} e^{-\frac{\tau}{M a D a}}+\sqrt{\frac{1}{M a D a}} \operatorname{erf}\left(\sqrt{\frac{\tau}{M a D a}}\right)\right] \\
& -\alpha\left[\phi_{3}(\eta, \tau)-\phi_{3}(\eta, \tau-1) H(\eta-1)\right],
\end{aligned}
$$

where

$$
\begin{array}{r}
\phi_{3}(\eta, \tau)=-\frac{e^{\beta \tau}}{\beta^{2}}\left\{\sqrt{\frac{1}{M a D a}+\beta} \operatorname{erf}\left(\sqrt{\left(\frac{1}{M a D a}+\beta\right) \tau}\right)\right. \\
+\frac{1}{\sqrt{\pi \tau}} e^{-\left(\frac{1}{M a D a}+\beta\right) \tau}-\sqrt{\operatorname{Pr}(R a+\beta)} \operatorname{erf}(\sqrt{(R a+\beta) \tau})
\end{array}
$$




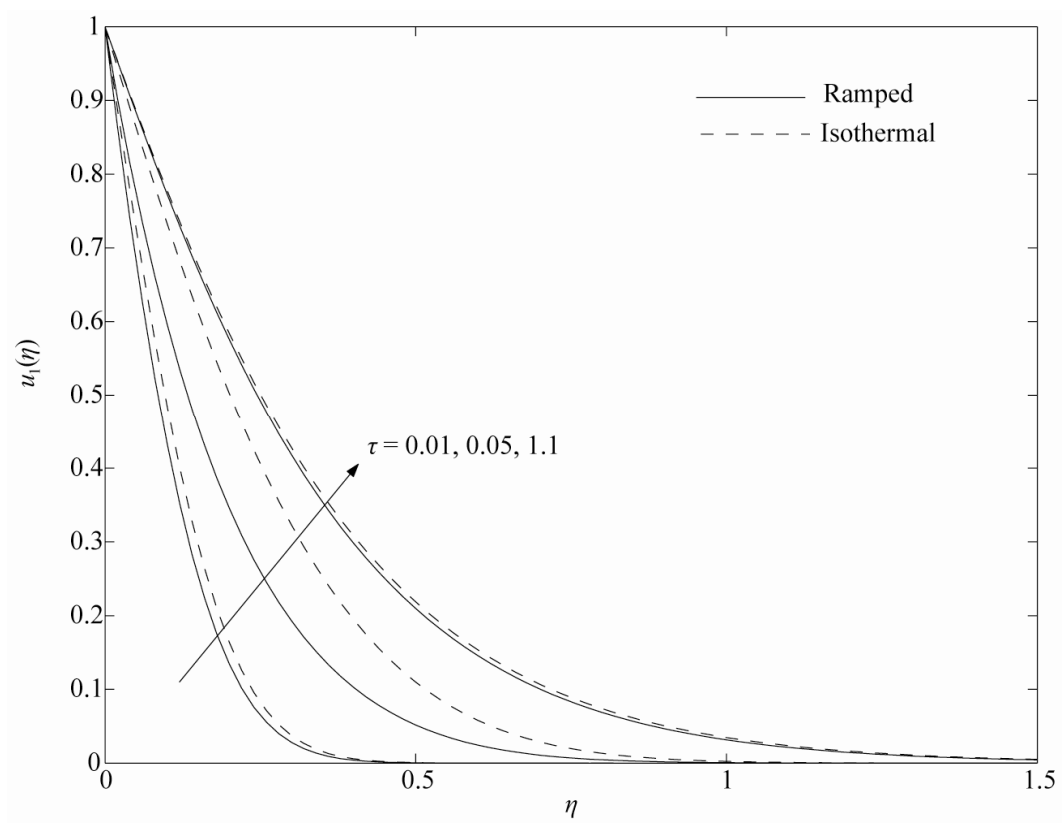

Figure 6. Velocity profiles for variations in time $\tau$ when $\operatorname{Pr}=0.71, G r=25, R a=2$ and $D a=0.04$.

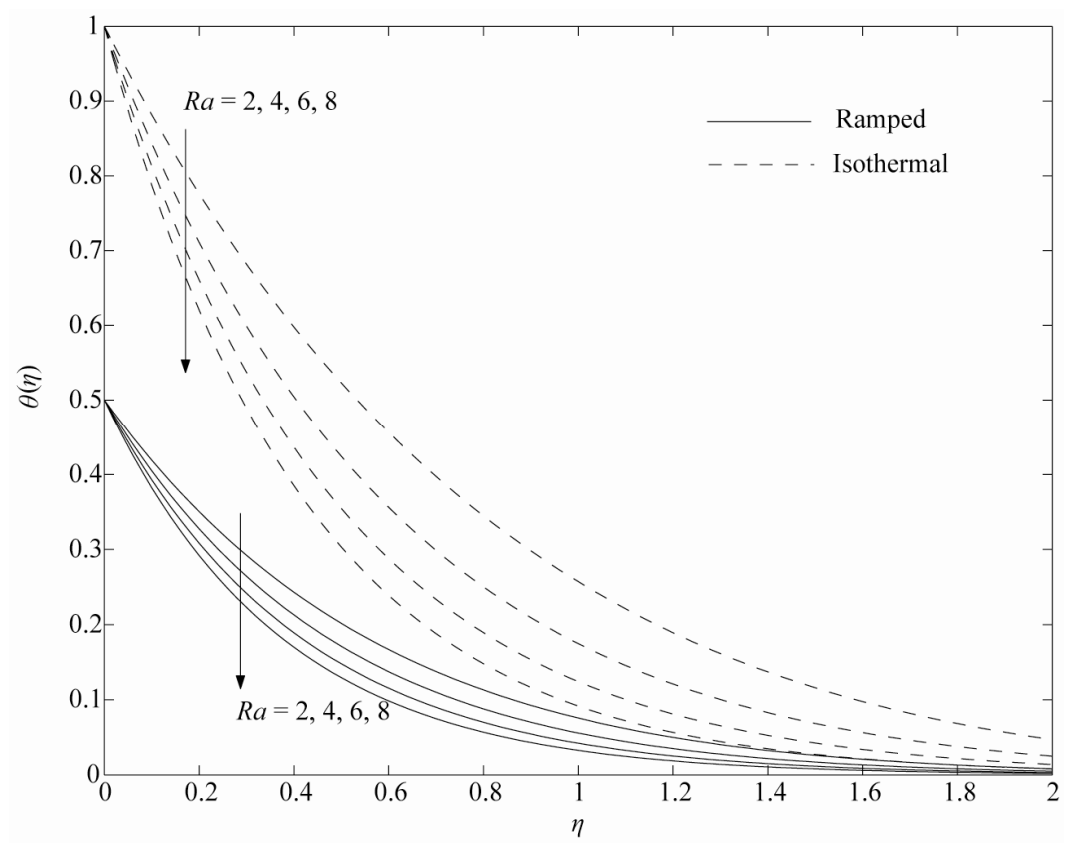

Figure 7. Temperature profiles for variations in $R a$ when $\operatorname{Pr}=0.71$ and $\tau=0.5$.

$$
\begin{array}{lr}
\left.-\sqrt{\frac{\operatorname{Pr}}{\pi \tau}} e^{-(R a+\beta) \tau}\right\}+\frac{1}{\beta}\left\{\frac{\sqrt{M a D a}}{2} \operatorname{erf}\left(\sqrt{\frac{\tau}{M a D a}}\right)\right. & \left.+\sqrt{\operatorname{Pr} R a}\left(\tau+\frac{1}{\beta}\right) \operatorname{erf}(\sqrt{R a \tau})+\sqrt{\frac{\operatorname{Pr}}{\pi \tau}}\left(\tau+\frac{1}{\beta}\right) e^{-R a \tau}\right\}, \\
+\frac{1}{\sqrt{M a D a}}\left(\tau+\frac{1}{\beta}\right) \operatorname{erf}\left(\sqrt{\frac{\tau}{M a D a}}\right) & \text { and for the isothermal wall temperature } \\
\left.+\frac{1}{\sqrt{\pi \tau}}\left(\tau+\frac{1}{\beta}\right) e^{-\frac{\tau}{M a D a}}\right\}-\frac{1}{\beta}\left\{\frac{1}{2} \sqrt{\frac{\operatorname{Pr}}{R a}} \operatorname{erf}(\sqrt{R a \tau})\right. & \left.N u=-\frac{\partial \theta}{\partial \eta}\right]_{\eta=0}=\sqrt{\operatorname{Pr} \operatorname{Ra}} \operatorname{erf}(\sqrt{\operatorname{Ra} \tau})+\sqrt{\frac{\operatorname{Pr}}{\pi \tau}} e^{-R a \tau}, \text { (37) }
\end{array}
$$




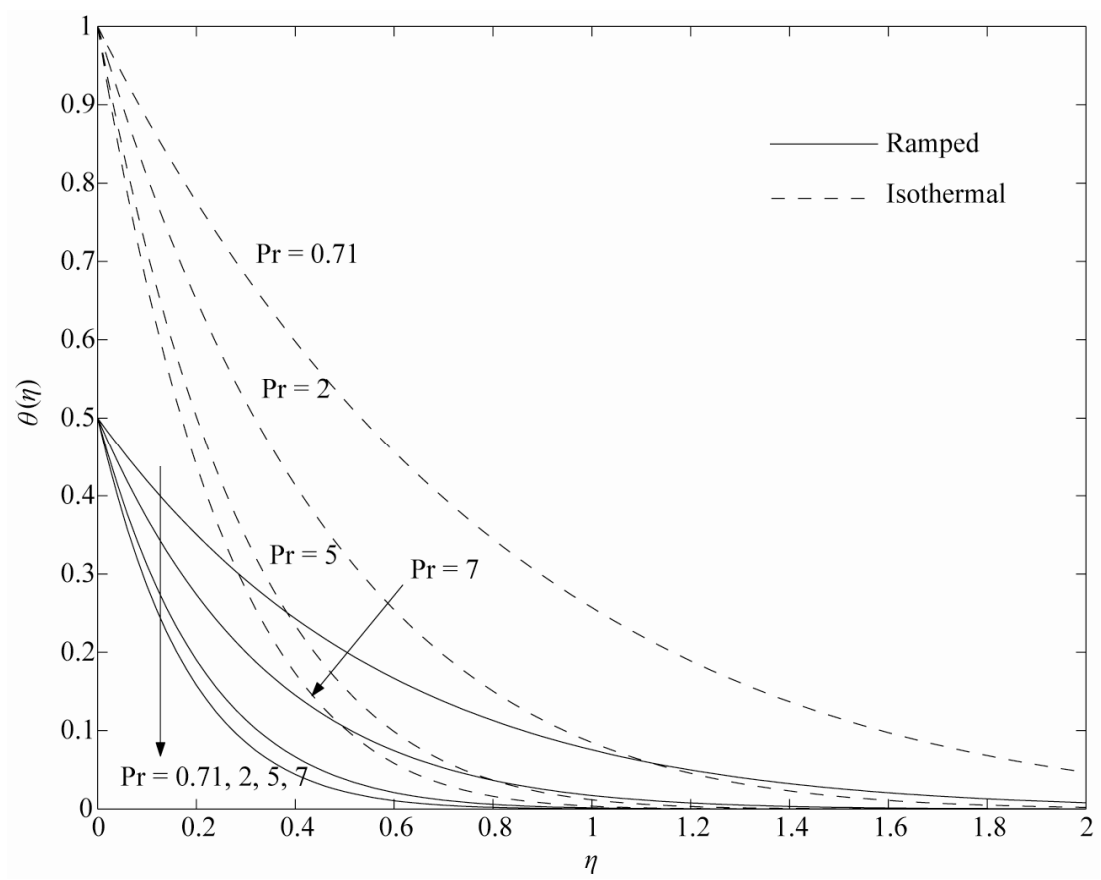

Figure 8. Temperature profiles for variations in $\operatorname{Pr}$ when $R a=2$ and $\tau=0.5$.

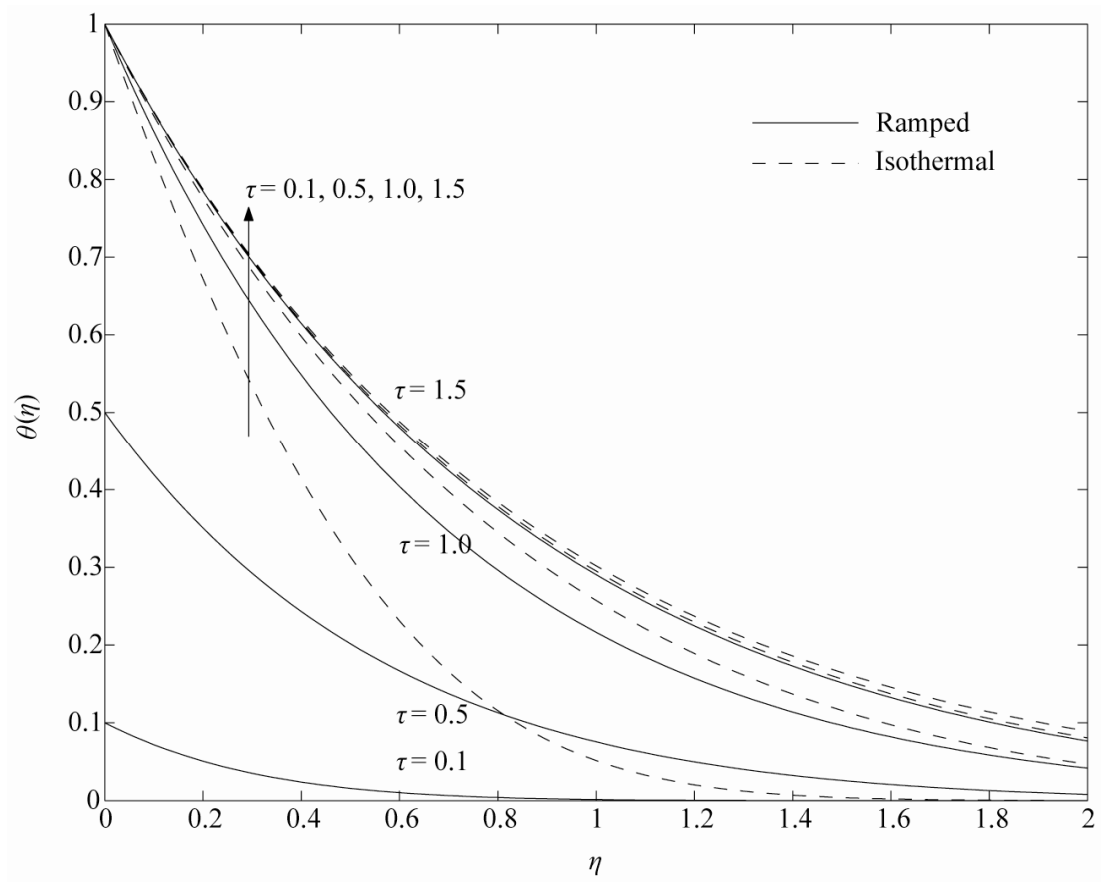

Figure 9. Temperature profiles for variations in time $\tau$ when $\operatorname{Pr}=0.71$ and $R a=2$.

$$
\begin{aligned}
& \left.\tau_{0}=\frac{\partial u_{1}}{\partial \eta}\right]_{\eta=0}=-\left[\left(1+\frac{\alpha}{\beta}\right) \frac{1}{\sqrt{M a D a}} \operatorname{erf}\left(\sqrt{\frac{\tau}{M a D a}}\right)\right. \\
& -e^{\beta \tau} \sqrt{\frac{1}{M a D a}+\beta} \operatorname{erf}\left(\sqrt{\left(\frac{1}{M a D a}+\beta\right) \tau}\right) \\
& +\frac{1}{\sqrt{\pi \tau}} e^{-\frac{\tau}{M a D a}}+\frac{\alpha}{\beta}\left\{e^{\beta \tau} \sqrt{\operatorname{Pr}(R a+\beta)} \operatorname{erf}(\sqrt{(R a+\beta) \tau})\right. \\
& -\sqrt{\operatorname{Pr} R a} \operatorname{erf}(\sqrt{R a \tau})\}] \text {. }
\end{aligned}
$$


Numerical results of shear stress at the wall $(\eta=0)$ are presented in Tables $\mathbf{1}$ to $\mathbf{4}$ for various values radiation parameter $R a$, Prandtl number $\mathrm{Pr}$, Grashof number $G r=4$, Darcy number $D a$ and time $\tau$. Table 1 shows that the magnitude of shear stress $\tau_{0}$ decreases for both ramped wall temperature as well as isothermal wall temperature with an increase in Darcy number $D a$ for fixed values of $R a$ and while the result is reversed with an increase in radiation parameter $R a$ for fixed values of $D a$. It is observed from Table 2 that the magnitude of shear stress $\tau_{0}$ decreases for both ramped wall temperature as well as isothermal wall temperature with increase in either Darcy number $D a$ or Grashof nubmer $\mathrm{Gr}$. It is also observed from Table 3 that the magnitude of shear stress $\tau_{0}$ decreases for both ramped wall temperature as well as isothermal wall temperature with an increase in time $\tau$. Table 4 displays that for creases for both ramped wall temperature as well as iso-

Table 1. Shear stress $-\tau_{0}$ for $\operatorname{Pr}=0.71, G r=10$ and $\tau=1$.

\begin{tabular}{ccccccc}
\hline & \multicolumn{3}{c}{ Ramped temperature } & \multicolumn{3}{c}{ Isothermal temperature } \\
\hline$D a / R a$ & 25 & 30 & 35 & 25 & 30 & 35 \\
0.040 & 3.93632 & 3.97912 & 4.01576 & 3.91459 & 3.95998 & 3.99849 \\
0.045 & 3.61771 & 3.66324 & 3.70195 & 3.59386 & 3.64215 & 3.68301 \\
0.050 & 3.34675 & 3.39462 & 3.43524 & 3.32075 & 3.37170 & 3.41473 \\
0.055 & 3.11244 & 3.16245 & 3.20487 & 3.08436 & 3.13779 & 3.18282 \\
\hline
\end{tabular}

Table 2. Shear stress $-\tau_{0}$ for $\operatorname{Pr}=0.71, R a=25$ and $\tau=\mathbf{1}$.

\begin{tabular}{ccccccc}
\hline & \multicolumn{3}{c}{ Ramped temperature } & \multicolumn{3}{c}{ Isothermal temperature } \\
\hline $\mathrm{Da} / \mathrm{Gr}$ & 10 & 15 & 20 & 10 & 15 & 20 \\
0.040 & 3.9363 & 3.4044 & 2.8726 & 3.91459 & 3.37188 & 2.82917 \\
0.045 & 3.6177 & 3.0696 & 2.5214 & 3.59386 & 3.03377 & 2.47368 \\
0.050 & 3.3467 & 2.7840 & 2.2214 & 3.32075 & 2.74506 & 2.16937 \\
0.055 & 3.1124 & 2.5366 & 1.9608 & 3.08436 & 2.49454 & 1.90471 \\
\hline
\end{tabular}

Table 3. Shear stress $-\tau_{0}$ for $\operatorname{Pr}=0.71$ and $G r=10$.

\begin{tabular}{cccccccc}
\hline \multicolumn{4}{c}{ Ramped temperature } & \multicolumn{3}{c}{ Isothermal temperature } \\
$\tau / R a$ & 25 & 30 & 35 & 25 & 30 & 35 \\
0.5 & 4.93191 & 4.93417 & 4.93632 & 3.96181 & 4.00034 & 4.03430 \\
1.0 & 4.80524 & 4.81177 & 4.81752 & 3.91696 & 3.96170 & 3.99986 \\
1.5 & 4.69611 & 4.70716 & 4.71677 & 3.91474 & 3.96007 & 3.99855 \\
2.0 & 4.58754 & 4.60313 & 4.61657 & 3.91460 & 3.95998 & 3.99849
\end{tabular}

Table 4. Shear stress $-\tau_{0}$ for $R a=25, D a=0.4$.

\begin{tabular}{ccccccc}
\hline & \multicolumn{3}{c}{ Ramped temperature } & \multicolumn{3}{c}{ Isothermal temperature } \\
\hline $\operatorname{Pr} / \tau$ & 0.5 & 1.0 & 1.5 & 0.5 & 1.0 & 1.5 \\
0.71 & 4.93191 & 4.80524 & 4.69611 & 3.96181 & 3.91696 & 3.91474 \\
2.0 & 4.95270 & 4.85151 & 4.76807 & 4.21229 & 4.17355 & 4.17170 \\
5.0 & 4.96972 & 4.88940 & 4.82698 & 4.41735 & 4.38361 & 4.38207 \\
7.0 & 4.97534 & 4.90190 & 4.84643 & 4.48504 & 4.45295 & 4.45151 \\
\hline
\end{tabular}

thermal wall temperature with an increase in Prandtl number $\mathrm{Pr}$.

Numerical results of the rate of heat transfer at the $(\eta=0)$ are presented in Tables 5 to 6 for various values radiation parameter $R a$, Prandtl number $\operatorname{Pr}$ and time $\tau$. Table 5 shows that the rate of heat transfer $\mathrm{Nu}$ increases for both ramped wall temperature as well as isothermal wall temperature with an increase in radiation parameter $R a$. Further, the rate of heat transfer increases for ramped wall temperature while it decreases isothermal wall temperature with an increase in time $\tau$ for fixed values of $R a$. It is observed from Table 6 that for fixed value of time $\tau$, the rate of heat transfer $N u$ increases for both ramped wall temperature as well as for isothermal wall temperature with an increase in Prandtl number $\operatorname{Pr}$.

\section{Conclusions}

An analysis is made to study the radiation effects on free convection flow past an impulsively started infinite vertical wall with ramped wall temperature in a porous medium. The velocity field and temperature distribution are presented for different physical parameters graphically. It is observed that the velocity profiles decrease with an increase in Prandtl number Pr for ramped wall temperature as well as isothermal wall temperature. An increase in Grashof number $\mathrm{Gr}$ leads to a rise in the values of velocity due to enhancement in buoyancy force. The velocity field is accelerated due to increase in Darcy number $D a$. The effect of the Prandtl number is very important in the temperature field. A fall in temperature occurs due to an increasing value of the Prandtl number. It is found that the temperature decreases as the radiation parameter increases for both ramped wall temperature as well as isothermal wall temperature. Further, the absolute value of shear stress $\tau_{0}$ increases for both ramped wall

Table 5. Rate of heat transfer $-\mathrm{Nu}$ for $\mathrm{Pr}=\mathbf{0 . 7 1}$.

\begin{tabular}{ccccccc}
\hline & \multicolumn{3}{c}{ Ramped temperature } & \multicolumn{3}{c}{ Isothermal temperature } \\
\hline$R a / \tau$ & 0.1 & 0.2 & 0.3 & 0.1 & 0.2 & 0.3 \\
2 & 0.32032 & 0.47976 & 0.61917 & 1.79436 & 1.46199 & 1.34228 \\
4 & 0.33924 & 0.53046 & 0.70774 & 2.06757 & 1.81588 & 2.08819 \\
6 & 0.35748 & 0.57787 & 0.78852 & 2.32489 & 2.13371 & 2.91893 \\
8 & 0.37509 & 0.62246 & 0.86297 & 2.56804 & 2.42239 & 2.39419 \\
\hline
\end{tabular}

Table 6. Rate of heat transfer $-N u$ for $R a=25$.

\begin{tabular}{ccccccc}
\hline \multicolumn{4}{c}{ Ramped temperature } & \multicolumn{3}{c}{ Isothermal temperature } \\
\hline $\operatorname{Pr} / \tau$ & 0.1 & 0.2 & 0.3 & 0.1 & 0.2 & 0.3 \\
0.71 & 0.32032 & 0.47976 & 0.61917 & 1.79436 & 1.46199 & 1.34228 \\
2.0 & 0.53761 & 0.80520 & 1.03919 & 3.01159 & 2.45375 & 2.25283 \\
5.0 & 0.85004 & 1.27314 & 1.64310 & 4.76174 & 3.87972 & 3.56204 \\
7.0 & 1.00578 & 1.50640 & 1.94414 & 5.63417 & 4.59055 & 4.21466 \\
\hline
\end{tabular}


temperature as well as isothermal wall temperature with an increase in Darcy number $D a$ for fixed values of $R a$ and while the result is reversed with an increase in radiation parameter $R a$ for fixed values of $D a$. The rate of heat transfer $\mathrm{Nu}$ increases for both ramped wall temperature as well as isothermal wall temperature with an increase in radiation parameter $R a$.

\section{References}

[1] P. S. Ghoshdastidar, "Heat Transfer," Oxford University Press, Oxford, 2004.

[2] D. A. Nield and A. Bejan, "Convection in Porous Media," Springer, New York, 2006, pp. 94-97.

[3] M. A. Mansour, "Radiation and Free Convection Effects on the Oscillating Flow past a Vertical Plate," Astrophysics and Space Science, Vol. 166, No. 2, 1990, pp. 269275. doi:10.1007/BF01094898

[4] A. Raptis and C. Perdikis, "Radiation and Free Convection Flow past a Moving Plate," Applied Mechanics and Engineering, Vol. 4, No. 4, 1999, pp. 817-821.

[5] U. N. Das, R. Deka and V. M. Soundalgekar, "Radiation Effect on Flow past an Impulsively Started Vertical Plate: An Exact Solutions," Journal of Applied Mathematics and Fluid Mechanics, Vol. 1, No. 2, 1966, pp. 111-115.

[6] R. Grief, I. S. Habib and J. C. Lin, "Laminar Convection of Radiating Gas in a Vertical Channel," Journal of Fluid Mechanics, Vol. 46, 1991, pp. 513-520. doi: $10.1017 / \mathrm{S} 0022112071000673$

[7] P. Ganesan and P. Loganathan, "Radiation and Mass Transfer Effects on Flow of an Incompressible Viscous Fluid past a Moving Vertical Cylinder," International Journal of Heat and Mass Transfer, Vol. 45, No. 21, 2002, pp. 4281-4288. doi:10.1016/S0017-9310(02)00140-0

[8] I. U. Mbeledogu, A. R. C. Amakiri and A. Ogulu, "Unsteady MHD Free Convection Flow of a Compressible Fluid past a Moving Vertical Plate in the Presence of Radiative Heat Transfer," International Journal of Heat and Mass Transfer, Vol. 50, No. 9-10, 2007, pp. 326-331. doi:10.1016/j.ijheatmasstransfer.2006.10.032

[9] O. D. Makinde, "Free-Convection Flow with Thermal Radiation and Mass Transfer past a Moving Vertical Porous Plate," International Communications in Heat and Mass Transfer, Vol. 32, No. 10, 2005, pp. 1411-1419. doi:10.1016/j.icheatmasstransfer.2005.07.005

[10] M. D. Abdus-Sattar and M. D. Hamid Kalim, "Unsteady Free-Convection Intraction with Thermal Radiation in a Boundary Layer Flow past a Vertical Porous Plate," Journal of Mathematical Physics, Vol. 30, 1996, pp.
25-37.

[11] C. Israel-Cookey, A. Ogulu and V. B. Omubo-Pepple, "Influence of Viscous Dissipation and Radiation on Unsteady MHD Free-Convection Flow past an Infinite Heated Vertical Plate in a Porous Medium with Time-Dependent Suction," International Journal of Heat and Mass Transfer, Vol. 46, No. 13, 2003, pp. 2305-2311. doi:10.1016/S0017-9310(02)00544-6

[12] K. D. Alagoa, G. Tay and T. M. Abbey, "Radiative and Free Convective Effects of a MHD Flow through a Porous Medium between Infinite Parallel Plates with Time-Dependent Suction," Astrophysics and Space Science, Vol. 260, 1999, pp. 455-468. doi:10.1016/S0017-9310(02)00544-6

[13] C. Israel-Cookey, E. Amos and C. Nwaigwe, "MHD Oscillatory Couette Flow of a Radiating Viscous Fluid in a Porous Medium with Periodic Wall Temperature," American Journal of Scientific and Industrial Research, Vol. 1, No. 2, 2010, pp. 326-331. doi:10.5251/ajsir.2010.1.2.326.331

[14] M. A. Sattar and M. A. Maleque, "Unsteady MHD Natural Convection Flow along an Accelerated Porous Plate with Hall Current and Mass Transfer in a Rotating Porous Medium," Journal of Energy, Heat and Mass Transfer, Vol. 22, 2000, pp. 67-72.

[15] M. A. Sattar, M. M. Rahman and M. M. Alam, "Free Convection Flow and Heat Transfer through a Porous Vertical Flat Plate Immersed in a Porous Medium," Journal of Energy Research, Vol. 22, No. 1, 2000, pp. 17-21.

[16] M. D. Abdus Samad and M. Mansur Rahman, "Thermal Radiation Interaction with Unsteady MHD Flow past a Vertical Porous Plate Immersed in a Porous Medium," Journal of Naval Architecture and Marine Engineering, Vol. 3, No. 1, 2006, pp. 7-14.

[17] N. C. Mahanti and P. Gaur, "The Effects of Varying Viscosity and Thermal Conductivity on Steady Free Convective Flow and Heat Transfer along an Isothermal Vertical Plate in the Presence of Heat Sink," Journal of Applied Fluid Mechanics, Vol. 2, No. 1, 2009, pp. 23-28.

[18] H. S. Takhar, P. Ganesan, K. Ekambavanan and V. M. Soundalgekar, "Transient Free Convection past a Semi Infinite Vertical Plate with Variable Surface Temperature," International Journal of Numerical Methods for Heat and Fluid Flow, Vol. 7, No. 4, 1997, pp. 280-296. doi:10.1108/09615539710165804

[19] A. C. L. Cogley, W. G. Vincenti and E. S. Gilles, "Differential Approximation for Radiative Heat Transfer in a Non Grey Gas near Equilibrium," American Institute of Aeronautics and Astronautics, Vol. 6, No. 3, 1968, pp. 551-553. 